\title{
Postprandiale Blutzuckerspitzen und KHK - Acarbose schützt nicht vor kardialen Spätfolgen
}

\author{
Eine spezifische Behandlung postprandialer Blutzu- \\ ckerspitzen schützt offenbar nicht vor kardialen \\ Spätfolgen. In der ACE-Studie ließ sich bei KHK-Pati- \\ enten durch Acarbose einzig die Entwicklung eines \\ Diabetes aufhalten. Das kardiovaskuläre Risiko blieb \\ von dieser Art der Intervention aber unbeeinflusst.
}

Entgleisungen postprandialer Blutzuckerspiegel lassen sich durch eine Therapie mit Acarbose zwar in den Griff bekommen. Kardiovaskuläre Spätfolgen verhindert eine solche Intervention aber offenbar nicht, wie sich in der randomisierten doppelblinden ACE-Studie gezeigt hat. Die Ergebnisse wurden auf dem EASD-Kongress in Lissabon vorgestellt und zeitgleich im „Lancet Diabetology \& Endocrinology“ publiziert.

Schon länger wird in Expertenkreisen diskutiert, ob es Sinn macht, bereits Patienten mit einer gestörten Glukosetoleranz mit einer antiglykämischen Substanz zu behandeln, die spezifisch postprandiale Blutzuckeranstiege verhindert, den Nüchternblutzucker aber kaum beeinflusst. Diese Art der Intervention soll der Entwicklung hin zu einem manifesten Diabetes und den damit einhergehenden Spätschäden entgegen wirken.

Das Konzept ging zumindest in der 2002 veröffentlichten STOP-NIDDM-Studie auf. Nicht nur der Übergang eines Prädiabetes zu einem Diabetes ließ sich durch die Gabe von Acarbose aufhalten bzw. verzögern. Überraschenderweise ging der Alpha-Glucosidase-Hemmer auch mit einem niedrigeren kardiovaskulären Risiko einher. Allerdings waren gerademal $47 \mathrm{~Pa}$ tienten von einem entsprechenden Ereignis betroffen, was die Aussagekraft des Ergebnisses deutlich abschwächt.

\section{Weniger Diabetesfälle...}

In der ACE-Studie sollte die potenziell präventive Wirkung von Acarbose nun an 6.522 chinesischen Patienten mit KHK und gestörter Glukosetoleranz geprüft werden. Während des mittleren Follow-up von fünf Jahren waren bei den Patienten, die Acarbose einnahmen, zwar deutlich weniger Diabetes-Fälle aufgetreten. Das Risiko wurde im Vergleich zur Placebo-Gruppe um $18 \%$ gesenkt.

Allerdings hatte die Therapie keinen Einfluss auf das kardiovaskuläre Risiko der Teilnehmer. So erlitten $14 \%$ der Patienten, die dreimal täglich $50 \mathrm{mg}$ Acarbose einnahmen, einen nichttödlichen Herzinfarkt oder Schlaganfall, verstarben an einer kardiovaskulären Erkrankung oder wurden wegen unstabiler Angina pectoris oder Herzinsuffizienz in ein Krankenhaus eingewiesen (kombinierter primärer Endpunkt). In der PlaceboGruppe waren $15 \%$ von einem solchen Ereignis betroffen. Auch die Gesamtmortalität blieb unverändert.

\section{...aber kein Einfluss auf kardiovaskuläres Risiko}

Trotz dieser ernüchternden Ergebnisse wollen die Autoren um Rury Holman von der University of Oxford Diabetes Trials Unit das Konzept der Therapie postprandialer Blutzuckerspitzen nicht vollkommen aufgeben. „Obwohl in der ACE-Studie kein direkter Effekt der Acarbose auf das kardiovaskuläre Risiko gezeigt wurde, sollte man eine potenziell indirekte Wirkung nicht abtun“, schreiben sie in der Publikation. Indem Acarbose die Entwicklung eines Diabetes bei KHK-Patienten verzögere, könne sich die Substanz womöglich langfristig auch auf das kardiovaskuläre Risiko positiv auswirken.

\section{Konzept nicht ganz aufgeben}

Auch nach Ansicht von Prof. Michael Nauck und Prof. Juris Meier vom St. Josef-Hospital in Bochum ist hier das letzte Wort

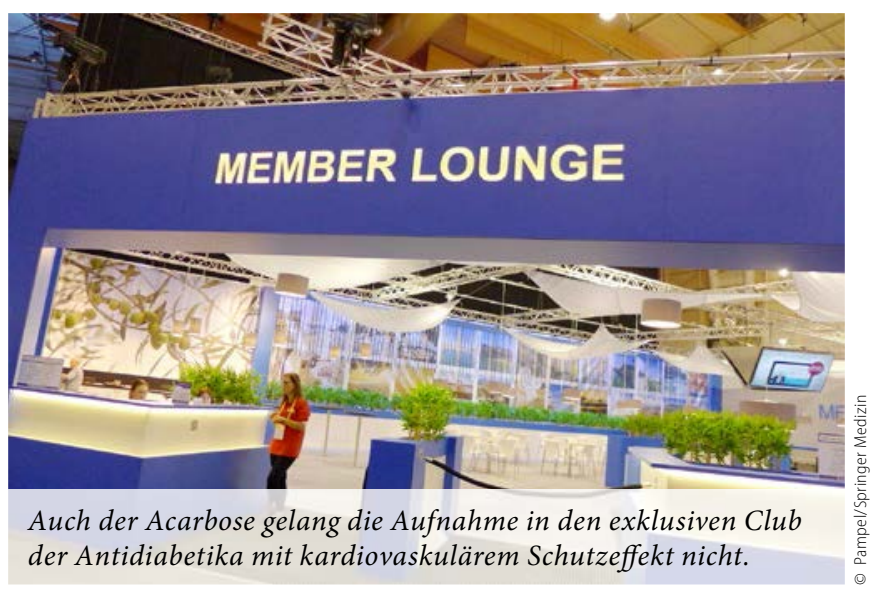

noch nicht gesprochen. Die Antwort auf die Frage, ob postprandiale Blutzuckerspitzen spezifisch das kardiovaskuläre Risiko erhöhen, müsse man nach der ACE-Studie wohl mit Nein zu beantworten. „Aber das gilt womöglich nicht für alle Patienten, etwa für solche, die sich bereits in einem fortgeschrittenem Diabetes-Stadium befinden“, führen die Diabetologen in einem Kommentar an. Für diese Patienten könnte eine Therapie mit Acarbose oder anderen Medikamenten, die den postprandialen Blutzucker beeinflussen, womöglich einen gewissen kardiovaskulären Schutz bieten. Dies müsse man in Studien prüfen. Allerdings weisen Nauck und Meier auch darauf hin, dass andere Maßnahmen wie intensive Lebensstiländerungen, Metformin oder Glitazone die Entwicklung eines Diabetes womöglich noch wirksamer aufhalten können.

Veronika Schlimpert

Referenzen:

1. Holman R, Coleman R, Chan J et al. Effects of acarbose on cardiovascular and diabetes outcomes in patients with coronary heart disease and impaired glucose tolerance (ACE): a randomised, double-blind, placebocontrolled trial Lancet Diabetes Endocrinol 2017; http://dx.doi. org/10.1016/ S2213-8587(17)30309-1

2. Nauck M, Meier J. Break point instead of ACE: acarbose, post-load glycaemic excursions, and cardiovascular events Lancet Diabetes Endocrinol 2017; http://dx.doi.org/10.1016/S2213-8587(17)30318-2 